\title{
Evaluation of phenotypic methods for rapid detection of Methicillin resistant Staphylococcus aureus in a tertiary care hospital
}

\author{
Samreen $\mathbf{S}^{1}$, Yadhav $\mathbf{K}^{2}$ \\ ${ }^{1}$ Dr Saba Samreen, Post Graduate Student, ${ }^{2}$ Dr Kala Yadhav M.L. Professor, both authors are attached with Department \\ of Microbiology, Bangalore Medical College and Research Institute, Karnataka, India.
}

Address for Correspondence: Dr Saba Samreen, Email: kysrz_sam5@yahoo.com

\begin{abstract}
Introduction: MRSA, an important pathogen causing nosocomial and community acquired infections, has posed a serious therapeutic challenge. A faster, cost effective method detecting MRSA is of utmost necessity. Objective: This study was done to look for the prevalence, antimicrobial sensitivity and evaluation of oxacillin E- test with chromogenic agar and cefoxitin disk diffusion methods for rapid detection of MRSA. Methods: A total of 100 isolates of Staphylococcus aureus isolated from clinical samples were included in the study. Antimicrobial susceptibility testing, oxacillin E-test, chromogenic agar inoculations were done as per CLSI guidelines. Results: Out of the 100 isolates, $46 \%$ were identified as MRSA by Cefoxitin disk diffusion method. All these isolates were detected by Chromogenic agar within 24hrs. All MRSA strains were resistant to penicillin (100\%). MRSA were found to be more multidrug resistant as compared to MSSA. All the strains were sensitive to Vancomycin, Linezolid irrespective of their methicillin status. Conclusion: Chromogenic agar was found to be rapid, easy, sensitive and specific method in detecting MRSA. It is ideal to inoculate sample directly onto Chromogenic agar in clinically suspected Gram positive infections or detected as Gram positive cocci in direct smear. The use of molecular methods for MRSA is largely restricted to reference laboratories and is not utilised in many laboratories as a routine tool. So, rapid and accurate detection of MRSA by Chromogenic agar helps to immediately start the antimicrobial therapy and avoid its spread.
\end{abstract}

Key words: Chromagar, Oxacillin E test, Rapid MRSA detection

\section{Introduction}

Globally, Staphylococcus aureus is considered as the most cause of nosocomial infections. It is a nonsporing bacteria that can survive in the environment under moist and dry conditions [1]. Methicillin resistant Staphylococcus aureus is an important pathogen causing nosocomial and community acquired infections. The first case of MRSA was isolated way back in 1961[2]. There has been a steady rise in the number of MRSA isolates which has evolved as a serious problem, since the resistance to methicillin indicates resistance to all $B$ lactam antibiotics [3]. MRSA strains harbor mecA gene which encodes a modified penicillin - binding protein (PBP2a), and they have low affinity for methicillin and all B-lactam antibiotics [4]. MRSA are being recognized as highly virulent and important human pathogens causing significant morbidity and mortality in hospitals

Manuscript received: $14^{\text {th }}$ August 2017

Reviewed: $24^{\text {th }}$ August 2017

Author Corrected: $2^{\text {nd }}$ September 2017

Accepted for Publication: $8^{\text {th }}$ September 2017 and community and are difficult to eradicate because of multidrug resistance [5]. A faster, cost effective method detecting MRSA is of utmost necessity. Approaches to rapid detection of MRSA include rapid culture methods and molecular techniques that can reduce the "turnaround time" for detection of MRSA colonization, leading to earlier isolation of colonized patients and lower rates of MRSA transmission. Most of the laboratories use Cefoxitin disk diffusion method for the routine testing of methicillin resistance. The gold standard method for antimicrobial susceptibility testing has been the minimum inhibitory concentration (MIC) that is determined by dilution methods or E strip method. In the recent years, MIC methods have been replaced by molecular methods that detect the mecA gene and are considered gold standard for determining the methicillin resistance in S. aureus [6]. The molecular methods for MRSA are not utilized in many laboratories as a routine tool. 


\section{Objectives}

This study was done to identify MRSA isolates, to detect the antimicrobial sensitivity pattern and also to evaluate and compare the usefulness and rapidity of

Oxacillin E -test with Chromogenic agar and Cefoxitin disk diffusion methods for rapid detection of MRSA.

\section{Materials and methods}

Study design: This is a cross sectional comparative study

Study period: From April 2016 to September 2016

Place of study: Department of microbiology in a tertiary care hospital, Bangalore

Inclusion criteria: All isolates of Staphylococcus aureus from various clinical samples like pus, blood, sputum, urine, exudates were included in the study.

Exclusion criteria: Gram negative isolates, coagulase negative Staphylococci, fungi

Sample size: A total of 100 isolates of Staphylococcus aureus isolated from various clinical samples were included in the study.

Sample collection and processing: Gram staining of samples was done. Samples yielding suspected gram positive cocci were inoculated onto Hi Media chocolate agar and Hi Media MeReSa chromogenic agar for MRSA. The isolates were identified as Staphylococci by standard biochemical techniques [7]. Strains growing on Chromogenic agar and yielding colonies with rose to mauve color were considered MRSA [Figure 1].
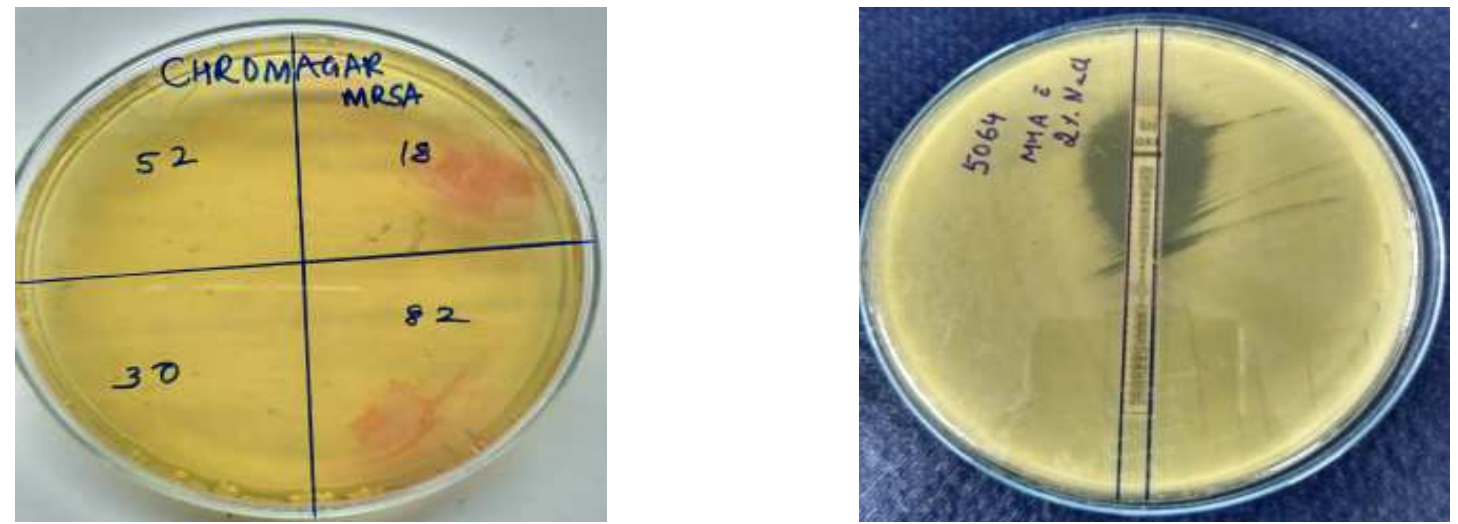

Figure-1: Rose-mauve colour colonies on Chrom Agar MRSA Figure-2: Oxacillin E strip on MHA with 2\% NaCl

Antimicrobial susceptibility testing using Kirby Bauer disk diffusion method and Oxacillin E-test was done as per CLSI guidelines [8, 9]. Known positive control MRSA (ATCC 29213) was included in each set. Antibiotics used were Penicillin (10mcg), Azithromycin(15mcg), Cefoxitin (30mcg), Linezolid (30mcg), Cotrimoxazole (23.75/1.25 mcg), Clindamycin (2mcg), Erythromycin $(15 \mathrm{mcg})$. Vancomycin sensitivity was tested using E strip using inoculum equivalent to 0.5 McFarland.

Oxacillin E- test was done using Mueller - Hinton agar supplemented with $2 \% \mathrm{NaCl}$ and an inoculum density equivalent to $0.5 \mathrm{McFarland}$ standard [ Figure 2]. Strains for which Oxacillin MIC was $>4 \mu \mathrm{g} / \mathrm{ml}$ were considered resistant. Oxacillin E -test MIC was our gold standard method. The sensitivity and specificity of other methods were compared with it.

\section{Results}

Out of the 100 isolates of Staphylococcus aureus, 46 were identified as Methicillin resistant and 54 as Methicillin sensitive by Oxacillin E-test. All these isolates were detected by Chromogenic agar by 18 to 24 hrs. No additional isolates were identified after 48hrs [Table 1]. 
Research Article

Table-1: Detection of MRSA by different methods.

\begin{tabular}{|c|c|c|c|}
\hline Bacterial isolate & Oxacillin E test (\%) & Cefoxitin disk diffusion (\%) & Chrom agar (\%) \\
\hline MRSA & 46 & 48 & 51 \\
\hline MSSA & 54 & 52 & 49 \\
\hline
\end{tabular}

Table-2: Comparative evaluation of various phenotypic methods used for MRSA detection.

\begin{tabular}{|c|c|c|c|c|c|c|c|c|}
\hline Test & $\begin{array}{c}\text { True } \\
\text { positive }\end{array}$ & $\begin{array}{c}\text { False } \\
\text { positive }\end{array}$ & $\begin{array}{c}\text { True } \\
\text { negative }\end{array}$ & $\begin{array}{c}\text { False } \\
\text { negative }\end{array}$ & $\begin{array}{c}\text { Sensitivity } \\
\mathbf{( \% )}\end{array}$ & $\begin{array}{c}\text { Specificity } \\
\mathbf{( \% )}\end{array}$ & $\begin{array}{c}\text { Positive } \\
\text { predictive } \\
\text { value (\%) }\end{array}$ & $\begin{array}{c}\text { Negative } \\
\text { predictive } \\
\text { value (\%) }\end{array}$ \\
\hline Oxacillin E-test & 46 & - & 54 & - & 100 & 100 & 100 & 100 \\
\hline $\begin{array}{c}\text { Cefoxitin disc } \\
\text { diffusion }\end{array}$ & 46 & 2 & 52 & - & 100 & 96.3 & 95.8 & 100 \\
\hline Chrom agar & 46 & 5 & 49 & - & 100 & 90.8 & 90.2 & 100 \\
\hline
\end{tabular}

Table-3: Showing comparison of antimicrobial resistance rates of MRSA \& MSSA.

\begin{tabular}{|c|c|c|}
\hline Antibiotic & Resistance in MRSA(\%) $\mathbf{n = 4 6}$ & Resistance in MSSA(\%) $\mathbf{n = 5 4}$ \\
\hline Penicillin & $46(100 \%)$ & $50(92 \%)$ \\
\hline Azithromycin & $14(30 \%)$ & $13(25 \%)$ \\
\hline Ciprofloxacin & $31(69 \%)$ & $25(47 \%)$ \\
\hline Linezolid & - & - \\
\hline Cotrimoxazole & $18(39 \%)$ & $15(28 \%)$ \\
\hline Clindamycin & $33(73 \%)$ & $31(57 \%)$ \\
\hline Erythromycin & $27(60 \%)$ & $26(49 \%)$ \\
\hline Vancomycin & - & - \\
\hline
\end{tabular}

Using the Cefoxitin disk diffusion method, 48 isolates were identified as MRSA. 2 isolates which were resistant to Cefoxitin 30mcg disk (zone size of $18-21 \mathrm{~mm}$ ) were found to be sensitive on Oxacillin E -test. Chromogenic agar detected 51 isolates as MRSA. 5 isolates were found to be sensitive on Oxacillin E -test. As Oxacillin was our gold standard, Cefoxitin disk diffusion method gave 2 false positives and chromogenic agar gave 5 false positives. Cefoxitin disk diffusion test had $100 \%$ sensitivity and $96.3 \%$ specificity whereas chromogenic agar had $100 \%$ sensitivity but a lesser specificity of $90.8 \%$. However, chromogenic agar gave results by 18 - 24hr. [Table 2].

All MRSA strains were resistant to penicillin (100\%). MRSA were found to be more multidrug resistant as compared to MSSA. All (100\%) the strains were sensitive to Vancomycin, Linezolid irrespective of their methicillin status [Table 3].

\section{Discussion}

According to our study, the prevalence of MRSA in our hospital was found to be $46 \%$. Other studies have also shown such a high MRSA prevalence in various parts of the country like $45.36 \%$ in a study by Loveena Oberoi [5], 40.6\% in a study by Muralidharan S[10], $54.85 \%$ in a study by Anupurba S[11] and 59.3\% in study by Tiwari HK [12].

However lower prevalence of $26.4 \%$ was reported in a study by Kumari N [13] and 19.5\% has been reported in a study by Tahnkiwale SS [14].
Chromogenic agar was found to be rapid and an easy method in detecting MRSA with a sensitivity of $100 \%$ and specificity of $90.8 \%$. A study by Somayeh Karami on chromogenic agar for MRSA showed $100 \%$ sensitivity and $97.9 \%$ specificity [6]. In a study by Loveena Oberoi it was found that the $47 \%$ isolates of Staphylococcus aureus were MRSA by cefoxitin disk diffusion, $46 \%$ by Chromagar for MRSA and $42 \%$ by Oxacillin E - test and also Chromagar was found to be less sensitive $(77.27 \%)$ \& less specific $(79.25 \%)$ in their study[5]. 
Our study showed that Cefoxitin disk diffusion method had a higher specificity compared to Chromogenic agar for MRSA but required a minimum of $48 \mathrm{hrs}$ for results. Whereas, chromogenic agar gave results by $18-24 \mathrm{hrs}$. It is ideal to inoculate sample directly onto chromogenic agar in clinically suspected Gram positive infections or detected as Gram positive cocci in direct smear, for the early detection of MRSA isolates and this will also enable early initiation of treatment. In recent years, MIC methods have been replaced by molecular methods which detect mecA gene as gold standard. However, the use of molecular methods for MRSA is largely restricted to reference laboratories and is not utilized in many laboratories as a routine tool. All the isolates were sensitive to vancomycin and linezolid. This is consistant with the results in study by Vidhani S et al [15].

\section{Conclusion}

There is a need for constant surveillance of MRSA and its antimicrobial profile. The hospital infection control policy and guidelines should be strictly implemented. Rapid and accurate detection of MRSA by Chromogenic agar helps to immediately start the antimicrobial therapy and avoid its spread.

Funding: Nil, Conflict of interest: None initiated, Permission from IRB: Yes

\section{References}

1. Kapil A, editor. Ananthanarayan \& Paniker. Text Book of Microbiology, $9^{\text {th }}$ ed. India: Universities Press Private Ltd.; 2014. p. 199-207

2. Jevons MP, Celbenin- Resistant Staphylococcus Br Med J 1961 1:124-25.

3. Santhanakumarasamy P.L, Thankian S Comparison of phenotypic and molecular methods in the detection of methicillin resistant Staphylococcus aureus and its implications J Sci Soc 2015; 42: 166-9.DOI: 10. 4103 / 0974-5009.165553

4. Velasco D et al. Evaluation of different methods for detecting methicillin resistance in S.aureus, J Antimicrob Chemother 2005;55(3):379-82 DOI: 10. 1093 / jac/dki017
5. Loveena Oberoi, Ramanpreet Kaur, Aruna Aggarwall. Prevalence and Antimicrobial susceptibility pattern of MRSA, International journal of applied biology and pharmaceutical technology

6. Karami.S , Rahbar M , Yousefi JV ,Evaluation of Five Phenotypic Methods for Detection of Methicillin Resistant Staphylococcus aureus (MRSA) .Iranian Journal of Pathology (2011)6 (1), 27 - 31

7. J G Collee; T J Mackie; J E McCartney, Practical Medical Microbiology ,Churchill livingstone, New York, 14th Edition

8. Bauer AW, Kirby WM, Sherris JC, Turck M. Antibiotic susceptibility testing by a standardized single disk method. Am J Clin Pathol. 1966 Apr;45(4):493-6.

9. Clinical and laboratory standards institute guidelines, CLSI 2016

10. Muralidharan S. Special article on methicillin resistant Staphylococcus aureus. J Acad clin microbial 2009; 11:15-16.

11. Anupurba S, Sen MR, Nath G, Sharma BM, Gulati AK, Mohapatra TM. Prevalence of methicillin resistant Staphylococcus aureus in a tertiary referral hospital in eastern Uttar Pradesh. Indian J Med Microbiol. 2003 Jan-Mar;21(1):49-51.

12. Tiwari HK, Sen MR. Emergence of vancomycin resistant Staphylococcus aureus (VRSA) from a tertiary care hospital from northern part of India. BMC Infect Dis. 2006 Oct 26;6:156.

13. Kumari N, Mohapatra TM, Singh YI. Prevalence of Methicillin-resistant Staphylococcus aureus (MRSA) in a Tertiary-Care Hospital in Eastern Nepal. JNMA J Nepal Med Assoc. 2008 Apr-Jun;47(170):53-6.

14. Tahnkiwale SS, Roy S, Jalgaonkar SV. Methicillin resistance among isolates of staphylococcus aureus: Antibiotic sensitivity pattern and phage typing. Ind J Med Sci 2002; 56:330-4.

15. Vidhani S, Mehndiratta PL, Mathur MD. Study of methicillin resistant Staphylococcus aureus isolates from high risk patients. Indian J Med Microbiol 2001;19:87-90.

\section{How to cite this article?}

Samreen S, Yadhav K. Evaluation of phenotypic methods for rapid detection of Methicillin resistant Staphylococcus aureus in a tertiary care hospital. Trop J Path Micro 2017;3(3):294-297.doi: 10.17511/jopm.2017.i3.11. 